\title{
Novel Semi-Passive RFID System for Indoor Localization
}

\author{
Akshay Athalye, Vladimir Savic, Miodrag Bolic and Petar M. Djuric
}

\section{Linköping University Post Print}

\section{Tweet}

N.B.: When citing this work, cite the original article.

C2013 IEEE. Personal use of this material is permitted. However, permission to reprint/republish this material for advertising or promotional purposes or for creating new collective works for resale or redistribution to servers or lists, or to reuse any copyrighted component of this work in other works must be obtained from the IEEE.

Akshay Athalye, Vladimir Savic, Miodrag Bolic and Petar M. Djuric, Novel Semi-Passive RFID System for Indoor Localization, 2013, IEEE Sensors Journal, (13), 2, 528-537. http://dx.doi.org/10.1109/JSEN.2012.2220344

Postprint available at: Linköping University Electronic Press http://urn.kb.se/resolve?urn=urn:nbn:se:liu:diva-81905 


\title{
A Novel Semi-passive RFID System for Indoor Localization
}

\author{
Akshay Athalye, Vladimir Savić, Miodrag Bolić, and Petar M. Djurić
}

\begin{abstract}
In this paper we present a novel semi-passive Radio Frequency Identification (RFID) system for accurate indoor localization. The system is composed of a standard Ultra High Frequency (UHF), ISO-18000-6C compliant RFID reader, a set of standard passive RFID tags whose locations are known, and a newly developed tag-like RFID component, which is attached to the items that need to be localized. The new semi-passive component, referred to as sensatag (sense-a-tag), has a dual functionality in that it can sense the communication between the reader and standard tags in its proximity and can communicate with the reader like standard tags using backscatter modulation. Based on the information conveyed by the sensatags to the reader, localization algorithms based on binary sensor principles can be developed. We conducted a number of experiments in a laboratory to quantify the performance of our system, including two real applications, one finding the exact placement of items on shelves and another, estimating the direction of item movement.
\end{abstract}

Index Terms-indoor localization, RFID, passive tags

\section{INTRODUCTION}

Radio Frequency IDentification (RFID) is a well-known technology for real-time identification of various assets and users. One of the main goals of RFID technology is to enable ubiquitous asset visibility. Precise determination of an asset location is of great importance in achieving this goal. Accurate localization using RFID can enable several applications such as location of tagged items in warehouses and location of assets and personnel in hospitals and offices [1], [2]. Stateof-the-art localization methods can be broadly classified into three categories [3], [4]:

1) distance-based methods

2) methods based on scene-analysis, and

A. Athalye is with the Center of Excellence of Wireless and Information Technology, Stony Brook University, USA, e-mail: akshay.athalye@cewit.stonybrook.edu; V. Savić was with the Signal Processing Applications Group, Universidad Politecnica de Madrid, Spain, and he is now with the Department of Electrical Engineering (ISY), Linköping University, Sweden, e-mail: vladimir.savic@liu.se; M. Bolic is with the School of Information Technology and Engineering, University of Ottawa, Canada, e-mail: mbolic@site.uottawa.ca; P. M. Djurić is with the Department of Electrical \& Computer Engineering, Stony Brook University, USA, e-mail: petar.djuric@stonybrook.edu.

This work was supported by the NSF SBIR grant IIP-0912774. V. Savic was supported by the FPU fellowship from the Spanish Ministry of Science and Innovation and partially supported by the ICT project FP7-ICT-2009-4248894-WHERE-2, CONSOLIDER-INGENIO 2010 under grant CSD200800010 COMONSENS, the Swedish Foundation for Strategic Research (SSF) and ELLIIT. P. M. Djurić was supported by the NSF under Award CCF1018323 and by the ONR under Award N00014-09-1-1154. The support of the Center of Excellence of Wireless and Information Technology at Stony Brook University is also very much appreciated.

Copyright (c) 2012 IEEE. Personal use of this material is permitted. However, permission to use this material for any other purposes must be obtained from the IEEE by sending a request to pubs-permissions@ieee.org.
3) proximity-based methods.

Distance-based methods rely on range measurements that can be Received Signal Strength (RSS), Time-Of-Arrival (TOA), or Time-Difference-of-Arrival (TDOA) [2], [5]. With such measurements at (at least three) different reference points and upon converting them to estimated distances, one can employ simple trilateration for localization. In [6], the authors propose indoor positioning system based on Kalman filtering (KF), where they use the measurements of the backscattered RSS signal propagated from nearby RFID tags. In [7], mobile robot localization is based on TOA of radio frequency signals, where the measured distances are used to compute the coordinates by Extended KF. Positioning can be improved by reducing the uncertainties in the measurements by using ultrasonic measurements [8], [9]. It is worth pointing out that a new localization method based on phase difference between two or more receiving antennas may provide a better accuracy and robustness than RSS-based methods [10].

Scene-analysis methods consist of two stages. First, environmental information (fingerprints) is acquired. Then, the target location is estimated by matching the measurements with the stored fingerprints, where the estimated position is the average of the $k$ closest matches. This method is used in the wellknown LANDMARC system [11], and later improved using virtual reference tags [12].

The main problem with the above methods is that they are affected by multipath distortions and by dynamic changes in the environment. One direction of investigation for resolving this problem is to work with proximity-based methods that exploit binary information, i.e., information about a target being within small ranges of reference tags. The location estimate is found either by associating the location of the target with that of the closest reference tag, or as the centroid obtained from the locations of all the reference tags that were detected by the target. This type of methods has already been used for localization of mobile robots or sensors with a large number of tags with known positions [13], [14], [16], [15].

Location of assets is a problem that was traditionally solved with active RFID and WiFi based technologies. These solutions require placing an active tag on assets and deploying a dense WiFi access point infrastructure. They become economically infeasible when tagging high volumes of low to medium cost items that are densely collocated. Estimating the location of an asset tagged with a standard passive or semipassive tag has been a much sought after application since the inception of RFID. There are some solutions based on technologies that exist on the market. Even though they are standard compliant, these solutions do not rely on off-the-shelf 
UHF readers and require directional antennas.

In this paper, we introduce a novel type of semi-passive Ultra High Frequency (UHF) RFID tag that has the capability to detect and decode backscatter signals from RFID tags in its proximity and to communicate this information to a standard RFID reader. We refer to this tag as sensatag (from sense-atag) [17], [18]. We show that when a sensatag is attached to an object that needs to be localized and the object is in an indoor environment that is populated with passive tags with known locations, one can estimate the location of the object with high accuracy. We performed many experiments in our laboratory so that we could quantify the performance of our system. More specifically, we studied two real applications. In one, the objective was determining the shelf where a tagged item was placed, and in another, estimating the direction of movement (DOM) of a tagged item.

The paper is organized as follows. In Section 2, we introduce our RFID system and the novel component, the sensatag. In Section 3, we present practical applications of our system. In Section 4, we describe the algorithms for sensatag localization and DOM estimation. The experimental results of our work that demonstrate the performance of the system are shown in Section 5. We conclude the paper with some final remarks in Section 6.

\section{A NOVEL TYPE OF RFID COMPONENT}

\section{A. System architecture and functionality}

The architecture of the proposed sensatag based system is shown in Fig. 1. The new RFID system is composed of a personal computer (host) that runs the localization algorithms, an off-the-shelf ISO 18006-C (Gen 2) compliant RFID reader, multiple passive Gen 2 tags placed at known locations as shown, and sensatags attached to objects that we desire to locate. The vicinity or the detection range of the sensatag, i.e., the area around it where it detects backscatter from tags is shown in the figure by a dotted circle. The maximum range of detection of the sensatag depends upon the strength of the tag backscatter and the sensitivity of the sensatag. Within this limit, the range can be controlled by varying a threshold parameter on the sensatag. The locations of all the tags are recorded into the system prior to operation. As seen from the figure, two of the deployed tags are in the range of the sensatag. Consequently, the sensatag will detect backscatter from these two tags. The result of this detection is stored temporarily as binary information on the sensatag. Subsequently, the sensatags talk to the reader as if they were regular tags and convey the information of this detection to the reader. Based on this information about the proximity of certain tags to the sensatag, the location of the sensatag can be determined using techniques ranging from simple proximity based association to more complex ones like triangulation and maximum likelihood estimation.

This localization technique is more robust than conventional techniques because it relies on detection of backscatter in the close proximity of the tag rather than on measuring the phase or RSS of the backscatter signal at longer distances where it usually is corrupted by the wireless fading channel.

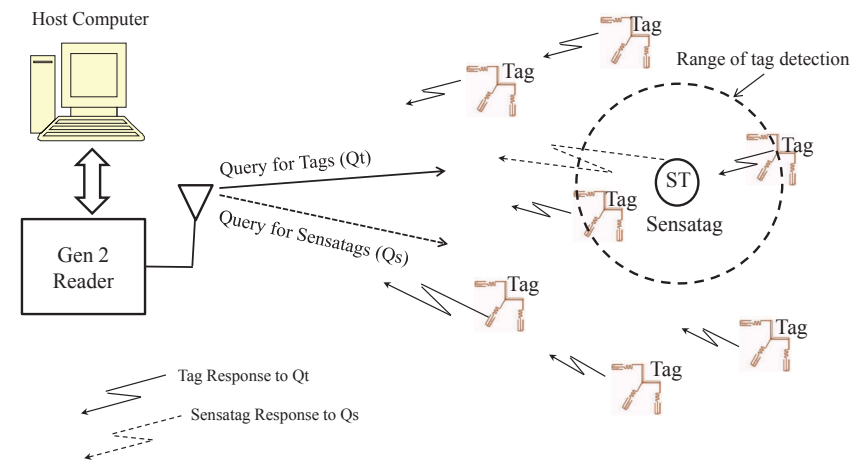

Fig. 1. Architecture of the sensatag-based RFID system.

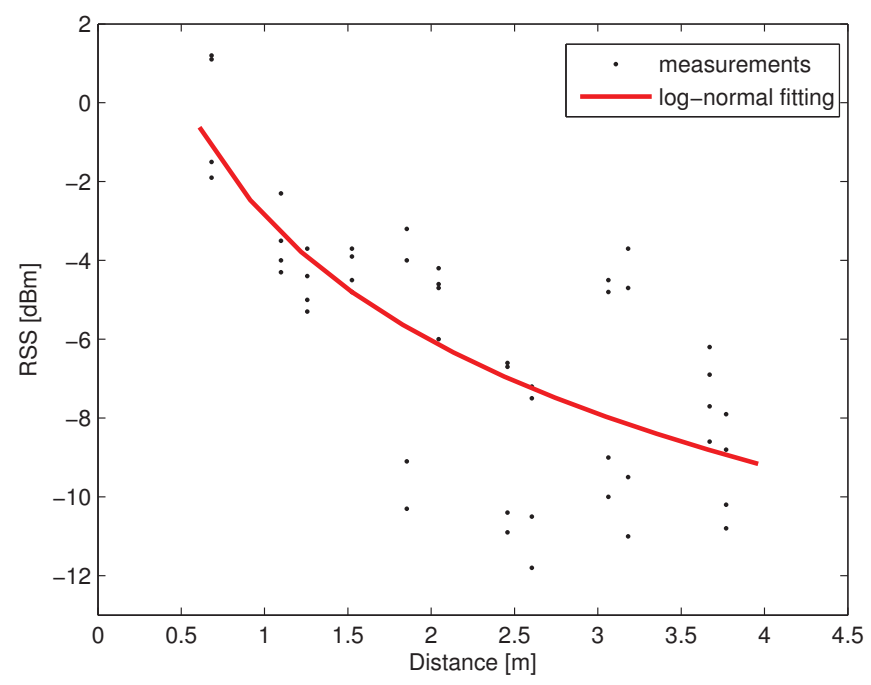

Fig. 2. Received signal strength (RSS) from the reader at different distances.

As example, in Fig. 2, we see experimental results of RSS measurements (in $\mathrm{dBm}$ ) obtained by the sensatag at various distances from the reader. The results show that, for a given distance, the RSS can vary considerably. This large variability makes the RSS-based methods susceptible to poor performance. As another example, with Fig. 3, we illustrate the sensing zone of the sensatag for two different positions of the reader. In order to obtain these measurements, a single sensatag was placed at $1.8 \mathrm{~m}$ and $3.6 \mathrm{~m}$ from the reader. The reader then sent out query signals and a single tag was placed in the vicinity of the sensatag. The tag was moved away until the sensatag could no longer detect the backscatter from the tag. The procedure was repeated by placing the tag at various angles in order to obtain the sensing zone of the sensatag. The results in Fig. 3 show that binary information (about the proximity of the tag) can be useful for our problem (see Section V, for detailed results).

The sensatags incorporate a special Gen 2 based locator protocol that allows them to function in harmony with standard 


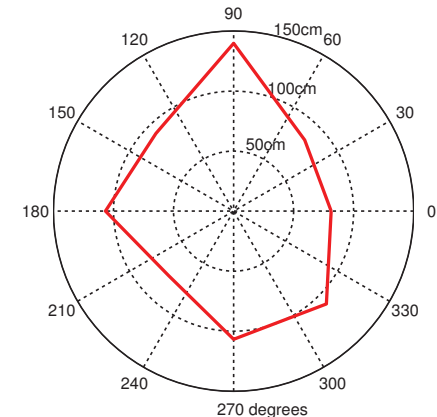

(a)

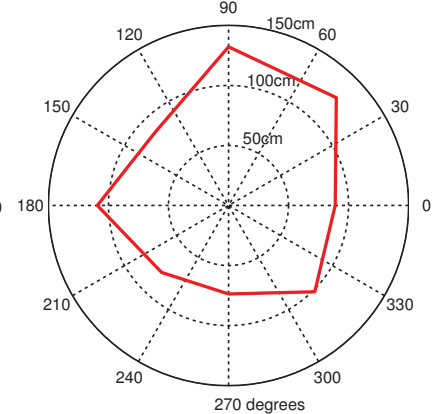

(b)
Fig. 3. Sensing zones of the sensatag: (a) $1.8 \mathrm{~m}$, (b) $3.6 \mathrm{~m}$, away from the reader. In the area outside the polygon marked by the red line, the probability of detection is zero.

readers and tags. As per this protocol, localization takes place through a two-stage query process, which is illustrated in Table 1. The host machine controls the reader to send out two distinct query signals, first a query $Q_{t}$ for tags, followed by query $Q_{s}$ for sensatags. These two distinct queries are generated using the Select mechanism provided by the Gen 2 standard. The sensatag operates in one of two states, listen or respond. A query of type $Q_{t}$ drives the sensatag into the listen state. During this query, the sensatag does not respond and instead listens for backscatter from tags in its vicinity. If it receives any such backscatter, it decodes this signal, extracts the tag's ID and stores it temporarily. The following query $Q_{s}$ drives the sensatag into the respond state. In this state, the sensatag responds to the reader query as if it were a standard tag, and conveys to the reader the information about which tags were detected in its vicinity during $Q_{t}$. This information is conveyed as part of the EPC ID of the sensatag. While the sensatag is in the listen state of a response to a $Q_{t}$ query, it decodes the tag IDs that it senses and stores a hash value corresponding to each sensed tag ID, along with a count indicating the number of times that it sensed that ID. Upon receiving a Query $Q_{s}$, all the hash values of the IDs that the sensatag detected, along with the respective counts are packed into a register known as the Located Tags ID (LTID) register. This register is prepended with a sensatag identifier and appended with a CRC-16. This collectively forms the sensatag EPC ID that is backscattered in response to a $Q_{s}$ query. This process of generation of the sensatag EPC ID is shown in Fig. 4. The reader sends this information to the host computer, which runs the algorithm for determining the location of the sensatag.

\section{B. Hardware Implementation}

A block diagram of the sensatag hardware is shown in Fig. 5. The sensatag communicates passively without an on board radio. An on board battery is used for powering up the sensatag circuitry, and thus, in its current form, the sensatag is a semi-passive device. The antenna is followed by a backscatter modulator, a conventional matching circuit and a Schottky diode-based detector. More details about the RF front-end are given in [18]. The output of the detector is fed to the analog section, the latter employing a comparator acting
TABLE I

FUNCTIONS OF THE HOST AND THE SENSATAG DURING COMMUNICATION AND PROCESSING PHASES

\begin{tabular}{|c|c|c|}
\hline & Host & Sensatag \\
\hline $\begin{array}{l}\text { Listening } \\
\quad\left(Q_{t}\right)\end{array}$ & $\begin{array}{l}\text { - Initiate query } Q_{t} \\
\text { - Obtain tags' IDs }\end{array}$ & $\begin{array}{l}\text { - Listening to the reader } \\
\text { - Detecting the tag } \\
\text { - Storing tag's ID }\end{array}$ \\
\hline $\begin{array}{l}\text { Reporting } \\
\left(Q_{s}\right)\end{array}$ & $\begin{array}{l}\text { - Initiate query } Q_{s} \\
\text { - Obtaining sensatag's } \\
\text { data }\end{array}$ & $\begin{array}{l}\text { - Listening to the reader } \\
\text { - Backscattering its ID and } \\
\text { partial IDs of detected tags. }\end{array}$ \\
\hline Processing & $\begin{array}{c}\text { - Associating tags } \\
\text { with sensatags } \\
\text { - Localization algorithm } \\
\text { - Estimation of direction } \\
\text { of movement }\end{array}$ & \\
\hline
\end{tabular}

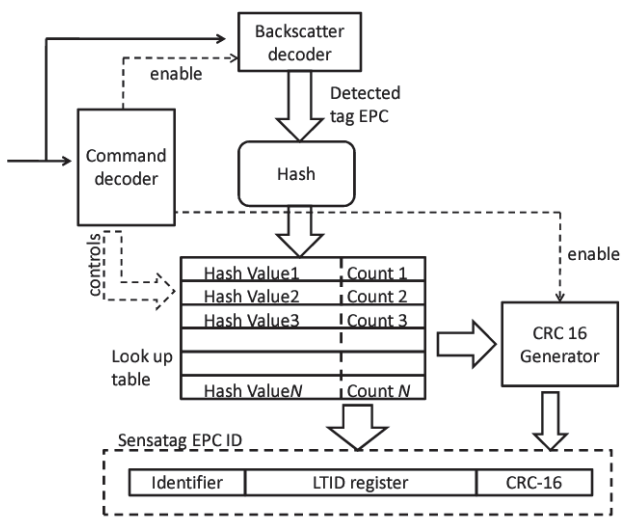

Fig. 4. Processing, storing, and generation of the sensatag EPC ID.

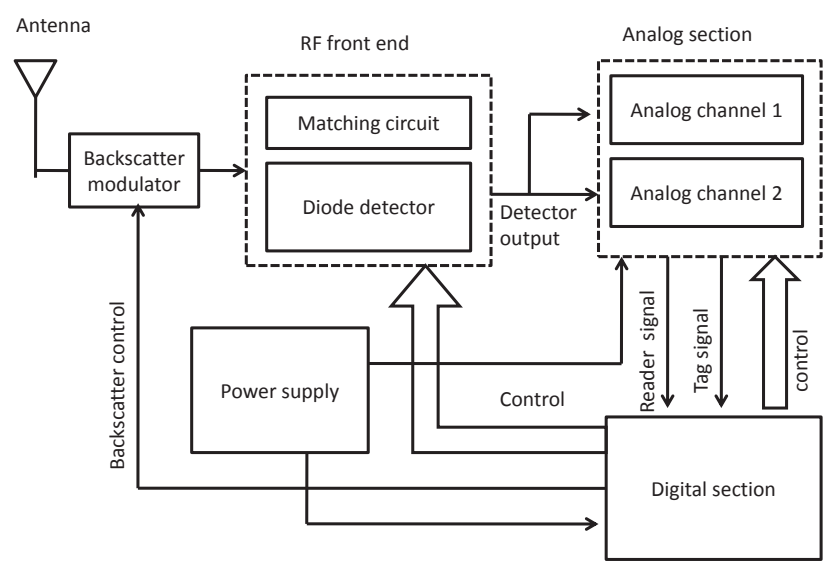

Fig. 5. Block diagram of the sensatag.

as a one bit $\mathrm{A} / \mathrm{D}$ converter. The output of the comparator is the input to the digital section, which implements the sensatag protocol.

Fig. 6 shows the signal received by the sensatag (at the output of the diode detector) in the vicinity of a backscattering UHF passive ISO 18000-6C compliant tag. For this experiment 


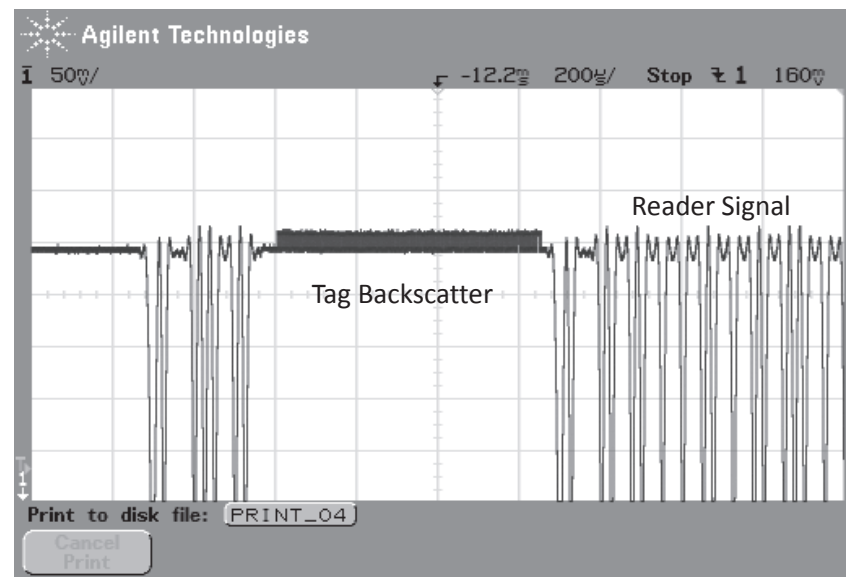

Fig. 6. Waveform of the signal captured by the sensatag.

a standard tag was stationed $1 \mathrm{~m}$ away from the assembled sensatag. The tag was queried by an Impinj Speedway reader situated $4 \mathrm{~m}$ from the tag. The waveform shows the detected signal prior to any analog processing. As seen from the figure, the amplitude, frequency and DC offset of the reader signal and the tag backscatter received at the sensatag are different. Hence the sensatag analog section should have the ability to adapt to the differences of these signals. The circuit for processing tag backscatter consists of a band-pass filter for removing the DC offset of the reader's continuous wave, followed by a comparator that is configured as a data slicer. The filter parameters and the threshold generation circuit for the comparator are adaptive. This is achieved by changing the values of the $\mathrm{RC}$ components used in the circuit using switches controlled by the digital section. The analog processing of the reader signal is exactly the same as in a standard passive tag.

The digital section runs state machines for ISO 18000-6C and for the sensatag protocols, and it performs partial baseband processing. In the current version, the digital section is implemented on an Xilinx Spartan 3AN FPGA chip. This device has an internal configuration memory resulting in significant space saving on the digital section of the board. The current implementation of the sensatag used in our experiments is shown in Fig. 7.

\section{Power Consumption and power management}

In its current implementation, the sensatag is a semi-passive device that is powered by an on board battery and needs a supply of $3.3 \mathrm{~V}$. In the current version of the design, some attention was paid to reduce the power consumption. However, the primary goal of this design was to implement and evaluate the novel functionality of the sensatag and not to reduce power consumption, particularly since the FPGA was used for rapid prototyping of the sensatag digital platform. Table II shows the current consumption of the individual blocks of the sensatag.

In order to conserve power, a sleep mode was implemented. The sensatag enters this mode when no querying signal is being received. As soon as the reader appears in the field, a

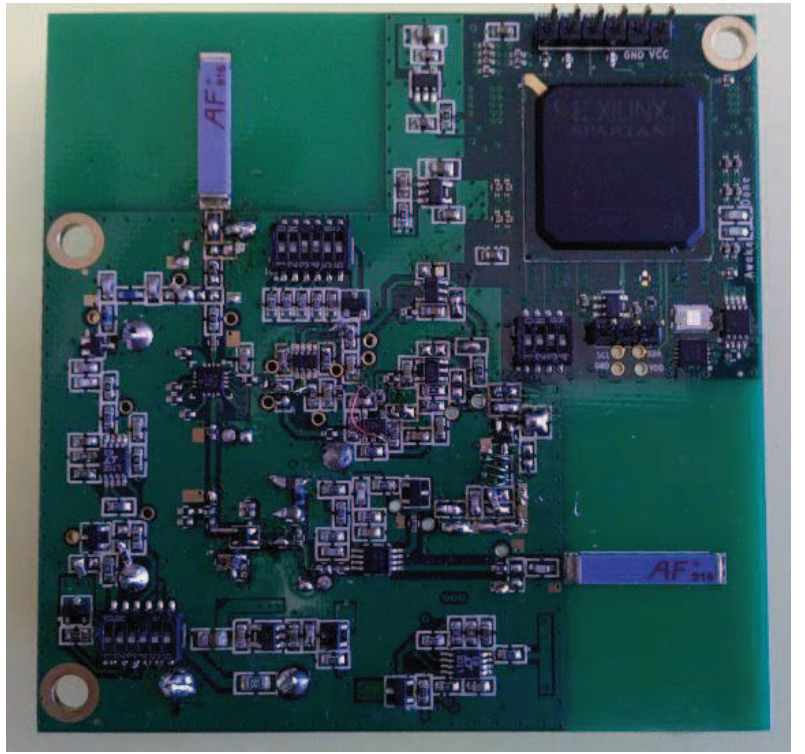

Fig. 7. The sensatag board (with dimensions $8.9 \mathrm{~cm} \times 8.9 \mathrm{~cm}$ ) used in the experiments.

TABLE II

CURRENT CONSUMPTION OF VARIOUS BLOCKS OF THE SENSATAG.

\begin{tabular}{|c|c|c|}
\hline Block & Supply Voltage & Current \\
\hline Backscatter Modulator & $2.5 \mathrm{~V}$ & $0.2 \mu \mathrm{A}$ \\
\hline Analog channel 1 & $3.3 \mathrm{~V}$ & $0.11 \mathrm{~mA}$ \\
\hline Analog channel 2 & $3.3 \mathrm{~V}$ & $3.1 \mathrm{~mA}$ \\
\hline Digital Section & $3.3 \mathrm{~V}$ & $24 \mathrm{~mA}$ \\
\hline
\end{tabular}

power detector in the front end senses this signal and issues a power on signal via the comparator that powers on the whole circuit. This significantly increases the battery life of the sensatag. Since the power consumption of the FPGA is high, in the next version of our design we will transfer part of the control logic to a low power micro-controller.

\section{PRACTICAL APPliCATIONS}

\section{A. Shelf Level Localization of tagged items}

Passive tags are often used in environments such as warehouses, retail stores and offices for tagging a large number of low-to-medium cost items. Frequently, the tagged items are densely co-located (especially on shelves in warehouse/retail store environments). An RFID reader used in these situations detects a large number of tags in its field of view and is unable to determine the specific location of each tag. Given the large area of warehouses and retail stores, use of stationary readers for inventorying items on all shelves is very expensive. A stationary reader based system would require a large number of readers and antennas and would necessitate a big deployment effort for mounting all the equipment and running power, network and antenna cables. The use of mobile RFID readers can make inventorying of items on shelves economically feasible. A single mobile reader with an integrated antenna, a battery and wireless connectivity can, for example, be mounted on a forklift and can be used to inventory items on all shelves in a warehouse. However, use of 
mobile readers further worsens location ambiguity since now there is a need to locate the reader within the warehouse and then locate a passive tag within the instantaneous field of view of the reader. By contrast, sensatags can provide very attractive solutions for accurate shelf-level localization of tagged items including solutions with just one reader.

In the environment described above, a sensatag can be affixed to each shelf or bin location. The sensatag will be programmed with an identifier corresponding to the location to which it is attached. In addition, a Gen 2 UHF passive (or semi-passive) tag will be attached to each asset (box, case or pallet) stored in the shelf or bin. We would like to point out here that an equivalent system can be constructed by affixing passive RFID tags with known pre-programmed location identifiers on each shelf and attaching sensatags to the objects placed on the shelves. The operation of both configurations of the system is essentially equivalent. Users can decide which configuration to use based on the relative number of shelf locations and items in their use case.

With such a system, inventory can be done by mobile readers which may be mounted, for example, on mobile carts, forklifts or in some cases may even be handheld. As the mobile reader moves through an isle, it reads the ID's of all the tagged items. Each sensatag senses backscattering tags in its own vicinity and reports this information to the reader in accordance with the protocol described in the previous section. Using the information obtained from the sensatags, the system can determine the shelf location of each item (e.g., book in a library).

\section{B. Direction of Movement of tagged items through portals}

Storage facilities often inventory their assets as the assets enter and leave them by installing RFID portals at each entry/exit point (commonly known as dock doors). In these environments, conventional RFID systems suffer from two important shortcomings: i) ambiguity in direction of asset motion (entering or exiting the facility) and ii) cross reads between adjacent portals. These problems significantly hamper the business intelligence derived from the deployed RFID system. Sensatags can readily solve the above mentioned problems. By deploying sensatags on either sides (outside and inside) of each adjacent portal, the system can determine the specific portal a tagged asset came through and if it came in or went out.

\section{DATA PROCESSING}

In the system presented in this paper, passive RFID tags are deployed at pre-defined locations within the environment where localization is to be performed. A sensatag is attached to the target of interest. The reader is programmed to send out alternating queries for the tags and sensatags using the Select functionality. The sensatag attached to the target operates using the described locator protocol and conveys binary information about the presence or absence of responding tags to the reader.

\section{A. Tag localization}

As a result of the queries, the host receives a list of sensatags and their associated tags at predefined time instants. The accuracy of the system depends on the density of the reference tags deployed at known locations. Our goal in this paper is to achieve satisfying localization accuracy without any calibration once the fixed tags are deployed (even in environments with dynamical changes). Therefore, we decided to use three simple localization methods that should work well in such circumstances. They are based on i) association, ii) centroids, and iii) weighted centroids.

Let there be $M$ reference (passive) tags placed at known two-dimensional positions, $x_{i}(i=1,2, \cdots, M)$ and one sensatag at unknown position $l$. A reference tag can be detected by a sensatag with probability $p_{i}$. This probability depends on various factors, but primarily on the distance between the reference tag and the sensatag, orientation, and the power of the reader. This probability is easily estimated by counting the number of detections of a tag by a sensatag in a fixed number of reader queries.

With association we simply associate the sensatag with the nearest passive tag. The proximity is measured by comparing the $p_{i} \mathrm{~s}$ of each reference tag. The main drawback of association is that when more passive tags are detected by the sensatag, the $p_{i} \mathrm{~s}$ may not correctly reflect the distance from the sensatag. For example, if the sensatag is close to tag A, and the power is sufficiently large so that both tag A and tag $B$ are detected the same number of times, the association of the tag becomes random and as a result, the position error is larger.

One simple way of building a more robust method is to implement averaging of the positions of all the passive tags that have been detected by the sensatag. In that case, the position of the sensatag can be computed by: $\widehat{l}=\sum_{i} x_{i} / n$, where the summation is over the locations of the tags that have been detected, and $n$ is the total number of detected tags by the sensatags. Therefore, the estimated position will be the centroid of the positions of the detected passive tags. This approach does not take into account the number of detections.

A natural extension of the centroid method, is the weighted centroid (WC), where the estimated position is the weighted average of the positions of the detected tags. Since it is expected that the closer tags will be detected more times than more distant ones, the weights are proportional to probabilities of detection of the tags. So, the estimated position is obtained by

$$
\widehat{l}=\sum_{i} \widehat{p}_{i} x_{i}=\sum_{i} \frac{n_{d}^{i}}{n_{q}} x_{i},
$$

where $n_{q}$ is the number of queries, and $n_{d}^{i}$ is the number of detections of tag $i$ by the sensatag.

\section{B. Direction of movement estimation}

In a number of applications, it is important to know the direction of movement (DOM) of the person or object close to some monitoring area. For example, in warehouse applications it is very important to determine if tagged goods are moving 
into or out of a warehouse. This problem can in principle be easily solved if we use two antennas and by measuring the times of detection of the tagged object from the signals of the antennas [21]. However, due to the small physical distances of the whole setup, the more distant antenna from the incoming object would "read" the tag basically at the same time as the nearer antenna, which would prevent easy determination of the DOM. Our solution for estimating the DOM of the sensatag relies on a reader with one antenna and on a number of reference tags. DOM can be found by recognizing the pattern of the estimates (e.g., found by WC described in Section IV-A)). One benefit of this method is easy estimation of the non-movement of the tracked object, as explained below.

More specifically, our goal is to find if the target is moving left, moving right or standing within the monitoring area. Thus, for the problem at hand, the localizations are onedimensional (1D). Given two 1D estimates of the position at two consecutive instants, $y_{t-1}$ and $y_{t}$ (found by WC), we define the estimate of the direction by

$$
\widehat{d}=\left\{\begin{array}{rl}
1, & \text { if } y_{t}-y_{t-1}>\epsilon, \quad\left({ }^{\prime}\right. \text { move right') } \\
0, & \text { if }\left|y_{t}-y_{t-1}\right| \leq \epsilon, \quad(\text { 'stand' }),^{\prime}, \quad \text { if } y_{t}-y_{t-1}<-\epsilon, \quad\left(\text { 'move left' }^{\prime}\right)
\end{array},\right.
$$

where $\epsilon$ is some small predefined value.

In order to minimize errors, it is prudent to accumulate as many estimates $\widehat{d}$ as possible. Thus, we also define the random variables $N_{r}, N_{s}$, and $N_{l}$, which represent the number of occurrences of $\widehat{d}=1, \widehat{d}=0$, and $\widehat{d}=-1$, respectively, in a set of $n_{a}$ trials and where $N_{r}+N_{s}+N_{l}=n_{a}$. Let $C$ be a conditioning event that can take one of three outcomes, $C_{r}=$ move right, $C_{s}=$ stand, and $C_{l}=$ move left. The random variables $N_{r}, N_{s}$, and $N_{l}$ for a given $C_{i}, i \in\{r, s, l\}$, are distributed according to the trinomial distribution, i.e.,

$$
N_{r i}, N_{s i}, N_{l i} \sim \frac{n_{a} !}{n_{r} ! n_{s} ! n_{l} !} p_{r i}^{n_{r}} p_{s i}^{n_{s}} p_{l i}^{n_{l}},
$$

where the probabilities $p_{r i}, p_{s i}$, and $p_{l i}$ represent the probabilities of $\widehat{d}=1, \widehat{d}=0$, and $\widehat{d}=-1$, given the conditioning event $C_{i}$, respectively. These probabilities are assumed known (they can be obtained offline). Since our RFID system provides $n_{a}$ estimates of which $n_{r}$ result in $\widehat{d}=1, n_{s}$ in $\widehat{d}=0$, and $n_{l}=n_{a}-n_{r}-n_{s}$ in $\widehat{d}=-1$, we can obtain the class by maximizing the likelihood (or a posteriori probability if the prior probabilities of the classes are equal), that is by

$$
\widehat{C_{i}}=\arg \max _{C_{i}}\left(p\left(n_{r}, n_{s}, n_{l} \mid C_{i}\right)\right) .
$$

It has already been shown that this approach minimizes the misclassification rate [22].

\section{EXPERIMENTAL RESULTS}

Here we provide details of the experiments for studying our system for localization and DOM estimation. For our experimentation, we used commercial off-the-shelf devices, viz.,

1) an Impinj Speedway reader [23], and

2) Impinj Monza 3 Thin Propellor and Alien Higgs 3 Squiggle RFID tags [24], [25].

On the other hand, the sensatag is a new device designed, fabricated, and assembled by us.

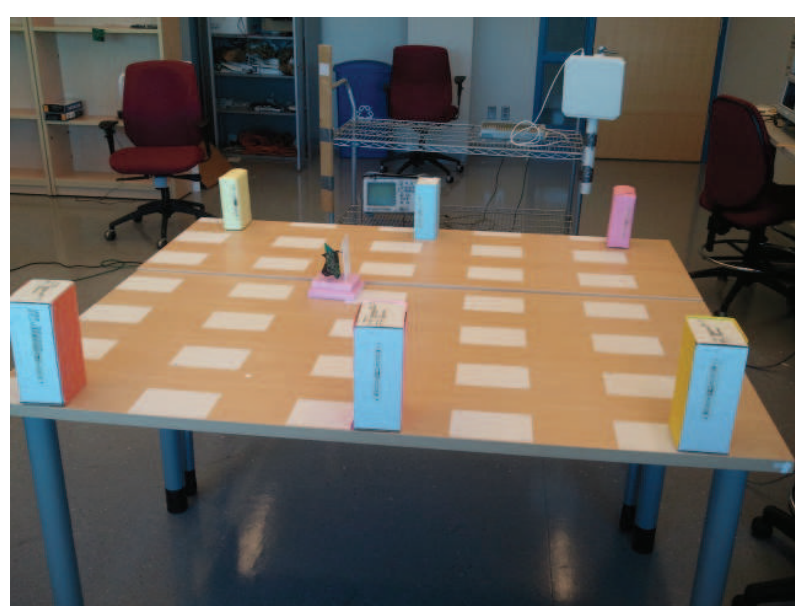

Fig. 8. Experimental setup. There were six reference points (shown by the standing boxes) each represented by two passive tags. There was one sensatag located in the middle. The reader and the antenna were in the background.

\section{A. Tag localization}

We deployed 12 passive tags at six reference points (we need a minimum of three reference points), where at each point we deployed two passive tags. The reason for having two passive tags per (nearly) same location was to prevent missing a tag by the sensatag because of eventual destructive superposition $^{1}$ of the signals from the reader and the tag. The overall area was $1.6 \mathrm{~m} \times 1.3 \mathrm{~m}$. The setup is shown in Fig. 8 . The difference between the reader antenna and the center of the plane in which the sensatag was located was $1.8 \mathrm{~m}$. The sensatag $^{2}$ was placed somewhere inside the area of interest. The objective was to estimate its position in the area.

In the first set of experiments, we studied the accuracy of the estimate as a function of reader power. We carried out localization of the sensatag at ten different positions and computed the average error (defined as the Euclidean distance between the true and the estimated position) as a function of power. The results are shown in Fig. 9. We see that the association method had the worst performance but was almost constant in the studied range of reader powers. The method based on the WC method outperformed the one that used the centroid. For both methods, the performance improved with the increase of reader power. The best performance of all the methods was by the weighted centroid with a reader power of $28 \mathrm{dBm}$ (the accuracy was about $14 \mathrm{~cm}$ ). If we keep increasing the reader power, a saturation effect decreases the performance.

In the next experiment, we studied the effect of the distance between the sensatag and the passive tags on the probability of detection. To that end, we acquired 20 independent measurements at 20 grid points. The results are shown in Fig. 10. We can see that the probability of detection can vary considerably even for the same distance. We, however, expect this variability; it is due to the different multipath components

\footnotetext{
${ }^{1}$ On the other hand, to avoid interference between tags, we also need to ensure minimal distance between tags. In our case, $10 \mathrm{~cm}$ was sufficient.

${ }^{2}$ In case of more sensatags, a minimal distance between them should be ensured (as for the passive tags). In that case, the detection will not be affected, but the query time will be increased (see Table 1).
} 


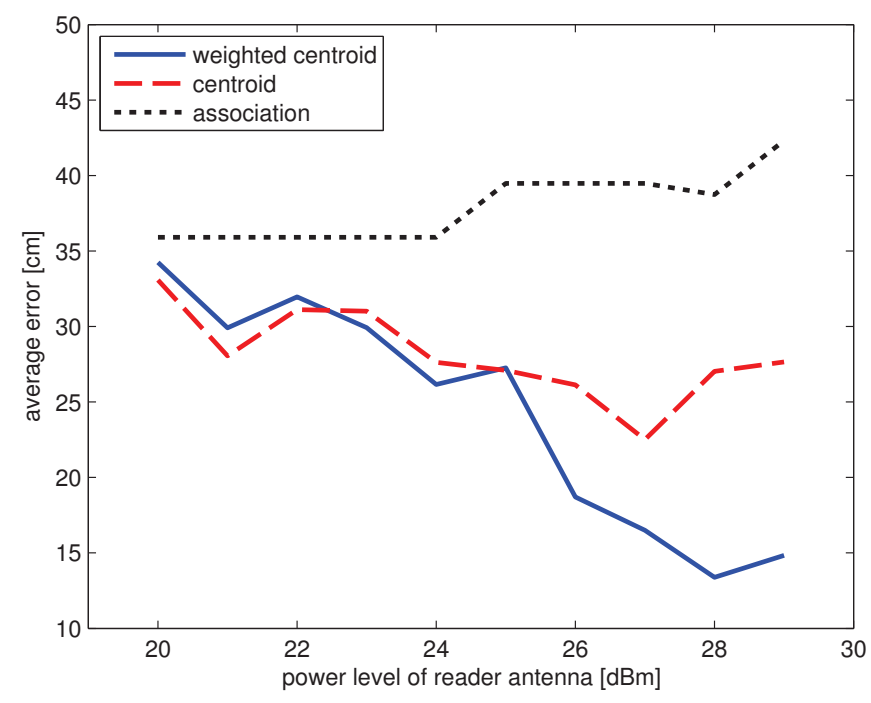

Fig. 9. The effect of reader power on the average position error of the three methods.

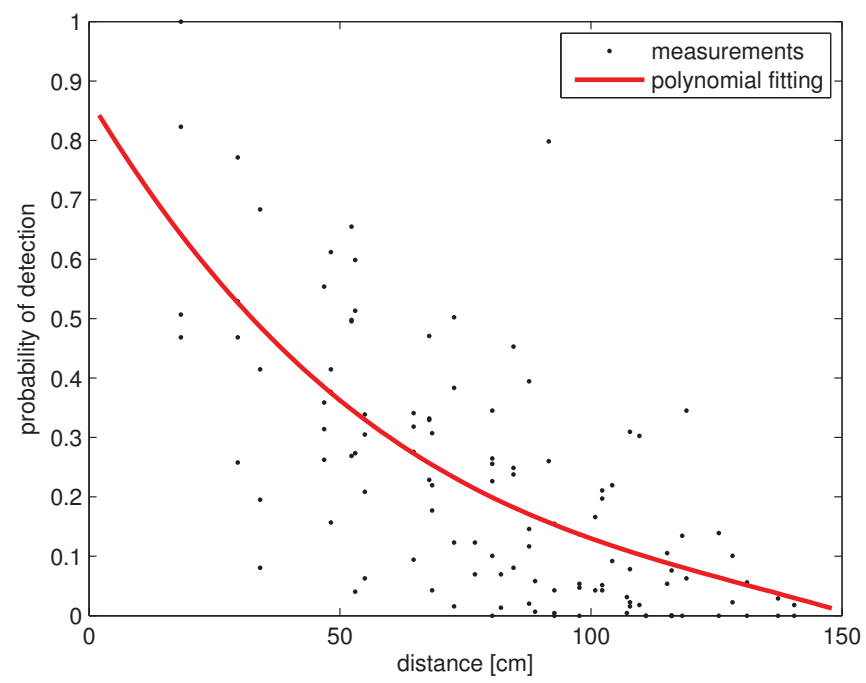

Fig. 10. Estimated probability of detection and the corresponding four-degree polynomial fitting.

and other factors that play role in formation of the signal received by the sensatag. We fitted the data with a four-degree polynomial function, which is also displayed in the figure. The curve shows how the probability of detection decreases monotonically with distance, which is the main motivation for using the WC method. Clearly, with weighting the locations of the detected passive tags, we give higher emphasis to the detected tags that are closer than the ones that are further away from the sensatag.

For comparison of the performance of the methods, we also used the empirical cumulative distribution function (CDF) of the location error of the three methods. The results are shown in Fig. 11. The CDFs of the errors confirm that the WC method performs significantly better than the other two methods. For example, the probability of the error being less than $40 \mathrm{~cm}$ is about 0.95 for the WC method, 0.82 for the centroid, and 0.4 for the association-based method.

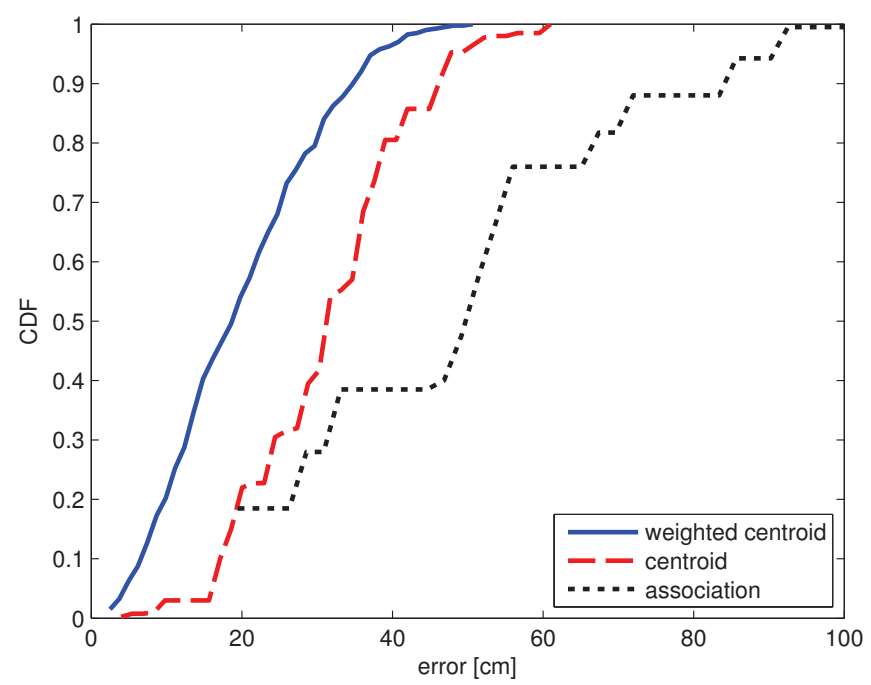

Fig. 11. Cumulative distribution function $(\mathrm{CDF})$ of position error for different algorithms.

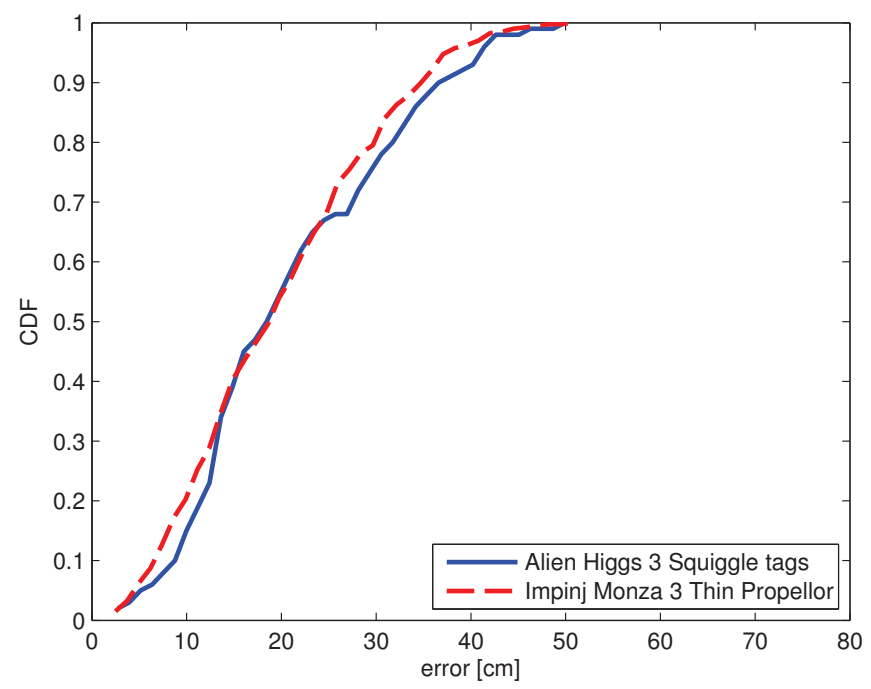

Fig. 12. CDF of position error for two different types of tags: the Impinj Monza 3 Thin Propellor and the Alien Higgs 3 Squiggle tags.

In the following set of experiments, we focused on the WC method and tested its robustness to environmental changes. We checked the accuracy of the method for different types of tags, different positions of the reader antenna, for LOS/NLOS scenarios, and different orientation of the sensatag antenna. We summarize the findings as follows:

- Different types of tags do not affect the accuracy (Fig. 12). For this experiment we used two types of tags: the Impinj Monza 3 Thin Propellor and the Alien Higgs 3 Squiggle tags.

- Different distance between the reader antenna and the center of deployment area affects the accuracy slightly (Fig. 13). However, this is valid under the assumption that the reader antenna provides sufficient power level for the whole deployment area (according to our tests, the minimum power level was $-15 \mathrm{dBm}$ ).

- The orientation of the plane of the sensatag antenna (we 


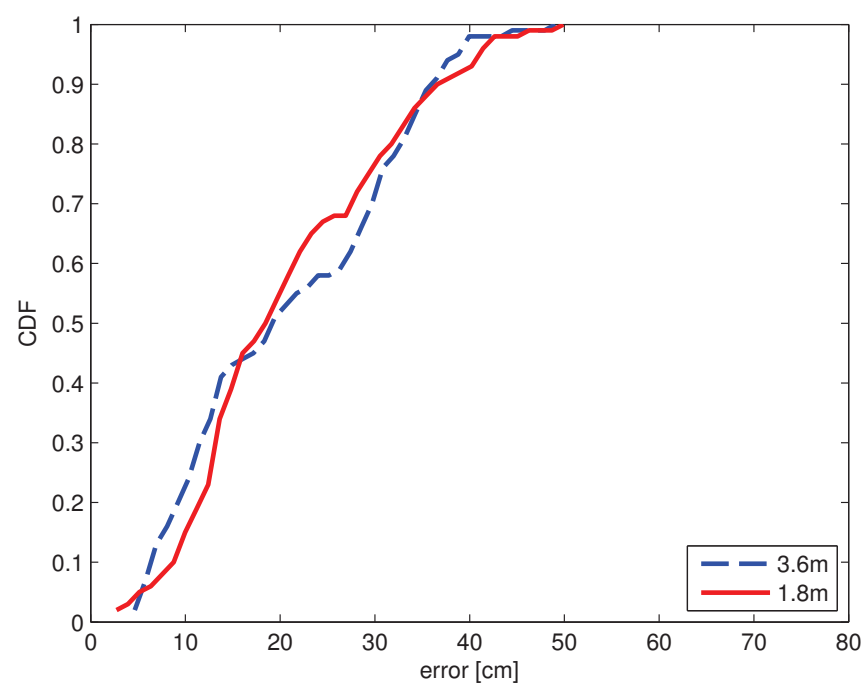

Fig. 13. CDF of position error for two different positions of the reader antenna.

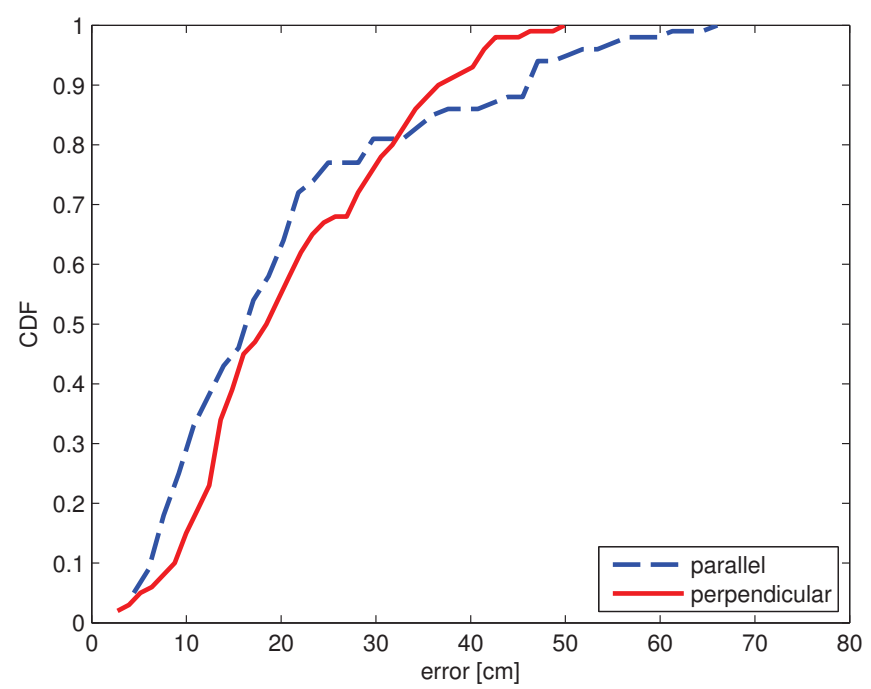

Fig. 14. CDF of position error for two different orientations of the sensatag antenna (w.r.t. the reader antenna). The two considered orientations represented the best and the worst case scenarios.

used Centurion Wireless patch) has significant impact on the accuracy. According to Fig. 14, the difference in error can be up to $15 \mathrm{~cm}$.

- Only metal and fluid obstacles affect the accuracy as expected (Fig. 15). The wooden shelf practically has no impact.

The above findings show that our method is robust to dynamic changes in the environment, so we expect that it can be used for a number of real applications. To illustrate it, we set up a scenario of a standard warehouse application where one needs to identify the shelf that has an item with an attached sensatag. The setup is shown in Fig. 16. In contrast to the previous scenario (in Fig. 8), the basis is vertical. In Fig. 17 we compare the CDFs of position error for a horizontal and vertical bases of the area, and see that in vertical basis the system provides better performance. This is achieved due to

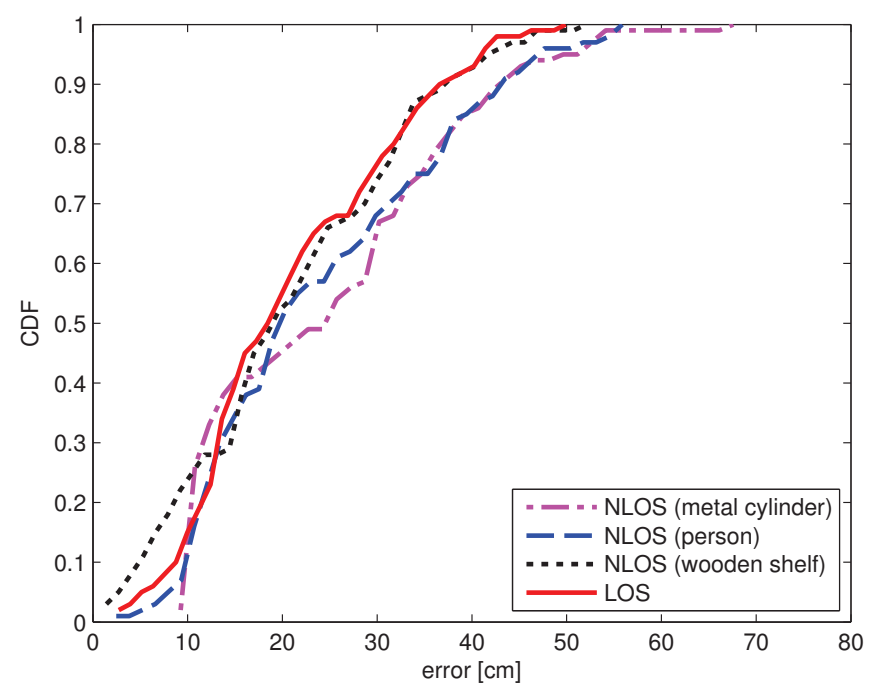

Fig. 15. CDF of position error for LOS and three NLOS scenarios.

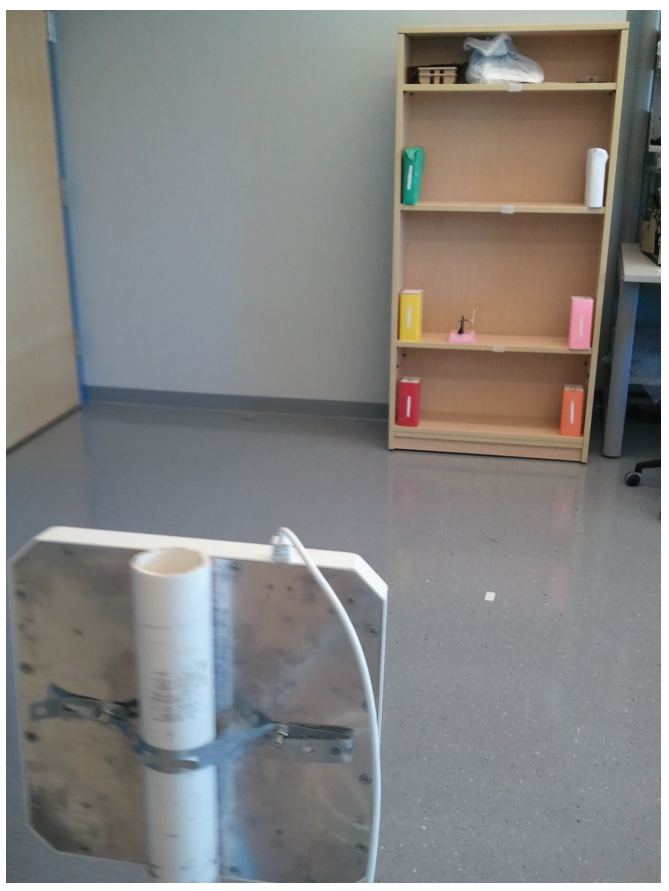

Fig. 16. Experimental setup for a typical warehouse application. The goal was to find the shelf where the sensatag was placed.

the higher density of the tags. We conducted 100 tests, and identified the correct shelf in all the trials.

\section{B. Direction of movement estimation}

In this set of experiments, we used a similar setup as for localization. The number of reference points was 9 (four placed on the left, and five placed on the right side of the area). The deployment area was $3 \mathrm{~m} \times 2 \mathrm{~m}$. In order to estimate the $p_{r i}, p_{s i}$, and $p_{l i}$, we moved the target sufficient number of times in an appropriate direction. For example, for the probabilities of moving to the right given that the tag was moving to the right, was standing, and was moving to the left, 


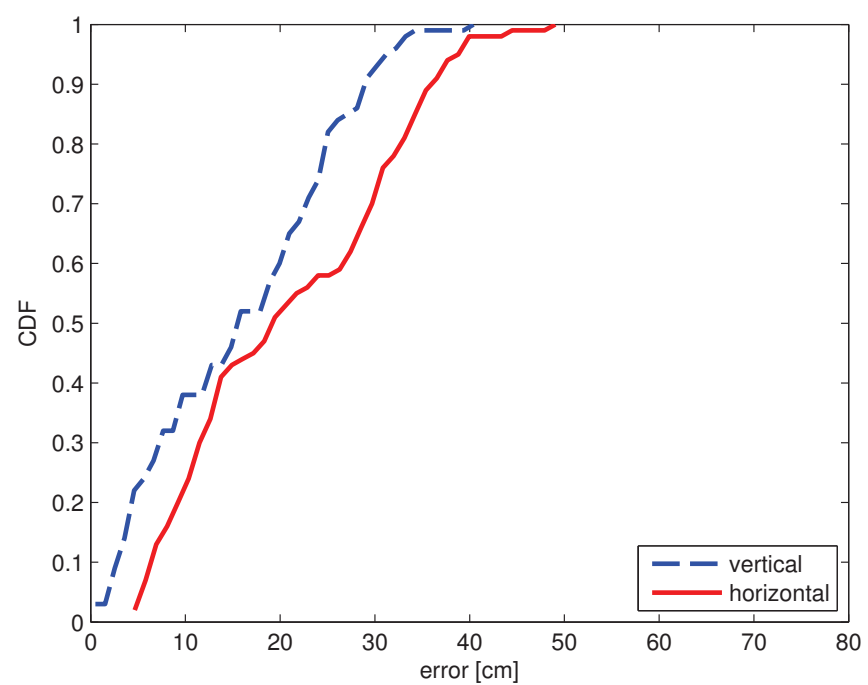

Fig. 17. CDF of position error for horizontal and vertical basis of the area

TABLE III

ACCURACY OF PROPOSED METHOD FOR DOM ESTIMATION

\begin{tabular}{c||c|c|c} 
& 'move right' & 'stand' & 'move left' \\
\hline \hline Target moves right & $90 \%$ & $10 \%$ & $0 \%$ \\
\hline Target stands & $0 \%$ & $95 \%$ & $5 \%$ \\
\hline Target moves left & $0 \%$ & $10 \%$ & $90 \%$
\end{tabular}

respectively, we obtained,

$$
\begin{aligned}
& \widehat{p}_{r r}=0.89 \\
& \widehat{p}_{r s}=0.53 . \\
& \widehat{p}_{r l}=0.09
\end{aligned}
$$

We can see that one single estimate is not sufficient for reliable DOM estimation. In order to test the method, we performed the experiments in which the target was moving left or right or was standing. The number of estimates (trials) was $n_{a}=$ 8. As we can see from Table III, we successfully estimated DOM in at least $90 \%$ of the test cases (out of 60 tests). The misclassification could not be avoided due to the overlap of the trinomial distributions. We can reduce misclassification if $n_{a}$ is larger so that the conditional distributions become tighter and with less overlap.

\section{Discussion: Localization in large areas}

Although our experiments provide us with important results about our RFID localization system, there is still the question of how it will perform in large areas and buildings. According to Fig. 11, we see that we get an average error of $30 \mathrm{~cm}$ or less for a transmit power of $21 \mathrm{dBm}$ or greater. The reader antenna had a gain of $6 \mathrm{~dB}$ and the tag antenna had a gain of $2 \mathrm{~dB}$. Under these circumstances, the received power in the "localization area" was about $-15 \mathrm{dBm}$. The system required a received power of greater than $-15 \mathrm{dBm}$ in all the area where localization was desired. The transmit power of commercial RFID readers is limited by FCC regulations to $30 \mathrm{dBm}$ (with a $6 \mathrm{~dB}$ ) antenna. Commercial readers have multiple antenna ports (2 to 4$)$. So, based on these results, we see that one reader can be used for localization in a circular area of radius $10 \mathrm{~m}$. Since a sensatag can reflect a greater amount of signal from its antenna than passive tags, the backscatter signal from the sensatag can reliably reach the reader at these distances. Therefore, for larger areas, we envision a deployment of multiple readers operating autonomously, with all the sensatag data gathered centrally for calculating location estimates.

\section{Vi. CONClusions}

In this paper we presented an UHF RFID system for accurate indoor localization of objects. The system is composed of (a) a number of passive tags whose locations are known and that serve as reference points, (b) a standard RFID reader, and (c) a new type of semi-passive tags called sensatags. The sensatags have dual ability (i) to detect and decode backscatter signals from RFID tags that are in their proximity and (ii) to communicate with the reader using backscatter modulation. When an object is tagged with a sensatag, it can be accurately located from the information acquired by the reader from the sensatag. We have conducted many experiments in which we studied the performance of our localization system. Some of the results are presented and show that the tagged object can be localized with high accuracy. Moreover, we showed how our system can be used for locating items (e.g., in warehouses), and direction of movement estimation (e.g., in retail stores). In future work, we plan to further reduce the cost and power consumption of the sensatag by replacing the FPGA on which the digital section is implemented with a microcontroller.

\section{REFERENCES}

[1] T. Sanpechuda, L. Kovavisaruch, "A review of RFID localization: Applications and techniques," in IEEE Proc. of International Conference on ECTI-CON, pp. 769-772, May 2008.

[2] A. H. Sayed, A. Tarighat, and N. Khajehnouri, "Network-based wireless location: Challenges faced in developing techniques for accurate wireless location information," Signal Processing Magazine, IEEE, vol. 22, no. 4, pp. 24-40, July 2005.

[3] M. Bouet, and A. L. Dos Santos, "RFID tags: Positioning principles and localization techniques," in IEEE Proc. of IFIP Wireless Days, pp. 1-5, Nov. 2008.

[4] Y. Zhang, X. Li, and M. Amin, "Principles and Techniques of RFID Positioning," in M. Bolić, D. Simplot-Ryl, I. Stojmenović, RFID Systems: Research Trends and Challenges, edited book, Wiley 2010.

[5] N. Patwari, J. N. Ash, S. Kyperountas, A. O. Hero III, R. L. Moses and N. S. Correal, "Locating the nodes: Cooperative localization in wireless sensor networks," Signal Processing Magazine, IEEE, vol. 22, no. 4, pp. 54-69, July 2005.

[6] S. S. Saab, and Z. S. Nakad, "A standalone RFID indoor positioning system using passive tags," in IEEE Transactions on Industrial Electronics, vol. 58, no. 5, pp. 1961-1970, May 2011.

[7] H. Cho and S. W. Kim, "Mobile robot localization using biased chirpspread-spectrum ranging," in IEEE Transactions on Industrial Electronics, vol. 57, no. 8, pp. 1-10, August 2010.

[8] B. S. Choi, and J. J. Lee, "Mobile robot localization scheme based on RFID and sonar fusion system," in IEEE Proc. of International Symposium on Industrial Electronics, pp. 1035-1404, July 2009.

[9] B. S. Choi, J. W. Lee, J. J. Lee, and K. T. Park, "A hierarchical algorithm for indoor mobile robot localization using RFID sensor fusion," in IEEE Transactions on Industrial Electronics, vol. 58, no. 6, pp. 2226-2235, June 2011.

[10] C. Hekimian-Williams, B. Grant, X. Liu, Z. Zhang, and P. Kumar, "Accurate localization of RFID tags using phase difference," in IEEE Proc. of International Conference on RFID, pp. 89-96, April 2010.

[11] L. M. Ni, Y. Liu, Y. C. Lau, and A. P. Patil, "LANDMARC: Indoor location sensing using active RFID," in IEEE Proc. of International Conference on Pervasive Computing and Communications, pp. 407-415, March 2003. 
[12] Y. Zhao, Y. Liu and L. M. Ni, "VIRE: Active RFID-based localization using virtual reference elimination," in IEEE Proc. of International Conference on Parallel Processing (ICPP), Sept. 2007.

[13] D. Niculescu and B. Nath, "Ad hoc positioning system (APS)," in IEEE GLOBECOM, vol. 5, pp. 2926-2931, Nov. 2001.

[14] P. M. Djurić, M. Vemula, and M.F. Bugallo, "Target tracking by particle filtering in binary sensor networks," in IEEE Transactions on Signal Processing, vol. 56, no. 6, pp.2229-2238, June 2008.

[15] W. Kim, K. Mechitov, J.-Y. Choi, and S. Ham, "On target tracking with binary proximity sensors," in IEEE/ACM Proc. of Information Processing in Sensor Networks (IPSN), pp. 301- 308, April 2005.

[16] S. Han, H. Lim, and J. Lee, "An efficient localization scheme for a differential-driving mobile robot based on RFID System," in IEEE Transactions on Industrial Electronics, vol. 54, no. 6, pp. 1-8, Dec. 2007.

[17] P. M. Djurić and A. Athalye, "RFID system and method for localizing and tracking a moving object with an RFID tag," Patent, Approved on: 2010-06-24; Application Number: 11799257, 2007.

[18] A. Athalye, V. Savić, M. Bolić, P. M. Djurić, "A radio frequency identification system for accurate indoor localization," in IEEE Proc of ICASSP, pp. 3028-3031, May 2011.

[19] D. M. Dobkin, The RF in RFID: Passive UHF RFID in Practice, Elsevier - Newnes, 2007.

[20] M. Bolić, A. Athalye, and T. Li, "Performance of passive UHF RFID systems in practice," in M. Bolic, D. Simplot-Ryl, I. Stojmenovic, RFID Systems: Research trends and challenges, edited book, Wiley 2010.

[21] Y. Oikawa, "Tag Movement Direction Estimation Methods in an RFID Gate System," in IEEE Proc. of International Symp. on Wireless Communication Systems, pp. 41-45, Sept. 2009.

[22] C. M. Bishop, Pattern Recognition and Machine Learning. Springer, 2006.

[23] Impinj, "The RFID Tag Antenna: Form Factor," Impinj RFID Technology Series, REV 1.0 8-05, 2005.

[24] Impinj, "Speedway Revolution Brochure," 2011 (available on http://www.impinj.com/Documents/Reader/Speedway_Revolution_ Reader_with_Autopilot/).

[25] Alien Technologies, "ALN-9640 Squiggle Inlay," 2012 (available on http://www.alientechnology.com/docs/products/ Alien-Technology-Higgs-3-ALN-9640-Squiggle.pdf).

Akshay Athalye received his $\mathrm{PhD}$. degree in electrical engineering from Stony Brook University, NY in 2007. Since 2008 he has been a Research Scientist at the Research Foundation of SUNY, Stony Brook, NY. His research interests are centered around Radio Frequency Identification (RFID) systems, with focus on various aspects such as signal processing, tag and reader hardware design, protocol development and localization techniques. In 2011, his research has led to the birth of a start-up company which provides proprietary RFID solutions to the Senior Care market. He has one patent, two pending patents and more than 15 research papers in the fields of RFID and signal processing. He has served as an external reviewer for various IEEE and ACM journals and conferences.

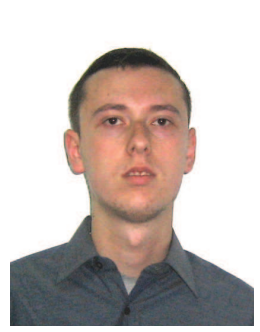

Vladimir Savić received his Dipl. Ing. Degree in electrical engineering from the University of Belgrade, Serbia, in 2006. From 2006 to 2008, he worked as Digital IC Design Engineer in Elsys Eastern Europe, Belgrade, Serbia. From 2008 to 2012, he worked as research assistant at Signal Processing Applications group, Universidad Politecnica de Madrid, Spain. He received his M.Sc. (in 2009) and his Ph.D degree (in 2012) in communications technologies and systems from the same university. During his Ph.D. studies, he spent three months as a visiting researcher at the Stony Brook University, NY, USA, and four months at the Chalmers University of Technology, Gothenburg, Sweden. In August 2012, he joined Communication Systems (CommSys) Division at Linköping University, Sweden as a postdoctoral researcher. His research interests include localization and tracking, message-passing algorithms, particle filtering, and distributed and cooperative signal processing.

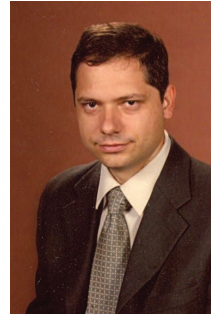

Miodrag Bolić received his Ph.D. degree in electrical engineering from the Stony Brook Univeristy, NY in 2004. From 1996 to 2000 he was a Research Associate with the Institute of Nuclear Sciences, Vinca, Belgrade, Serbia. Since 2004 he has been with the University of Ottawa, Canada where he is Associate Professor in the School of Electrical Engineering and Computer Science. Dr. Bolic's research includes biomedical signal processing and instrumentation, computer architectures, and wireless communications. He is a director of Computer Architecture, RFID and Medical Devices research groups at the University of Ottawa. He published about 35 journal papers, 4 book chapters and edited one book. He serves currently as an associate editor for the International Journal of Reconfigurable Computing, Hindawi and has been involved in organizing a number of conferences.

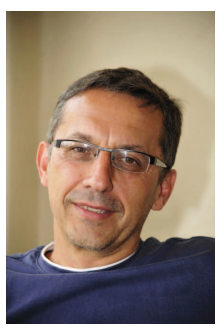

Petar M. Djurić received his B.S. and M.S. degrees in electrical engineering from the University of Belgrade, in 1981 and 1986, respectively, and his $\mathrm{Ph} . \mathrm{D}$. degree in electrical engineering from the University of Rhode Island, in 1990. From 1981 to 1986 he was a Research Associate with the Institute of Nuclear Sciences, Vinca, Belgrade. Since 1990 he has been with Stony Brook University, where he is Professor in the Department of Electrical and Computer Engineering. He works in the area of statistical signal processing, and his primary interests are in the theory of signal modeling, detection, and estimation and application of the theory to a wide range of disciplines. Prof. Djurić has been invited to lecture at many universities in the United States and overseas. In 2007 he received the IEEE Signal Processing Magazine Best Paper, and in 2008 he was elected Chair of Excellence of Universidad Carlos III de MadridBanco de Santander. During 2008-2009 he was Distinguished Lecturer of the IEEE Signal Processing Society. In 2012, Prof. Djurić received the EURASIP Technical Achievement Award. He has served on numerous committees for the IEEE, and currently he is a Member-at-Large of the Board of Governors of the Signal Processing Society. Prof. Djurić is a Fellow of IEEE. 